\title{
Continuity of Care Is Associated with Medical Costs and Inpatient Days in Children with Cerebral Palsy
}

\author{
Kuang-Tsu Yang ${ }^{1}$, Chun-Hao Yin ${ }^{2}$, Yao-Min Hung ${ }^{3,4,5}$, Shih-Ju Huang ${ }^{6}$, Ching-Chih Lee ${ }^{7,8}$ and \\ Tsu-Jen Kuo 9,10,11,* \\ 1 Division of Gastroenterology and Hepatology, Department of Internal Medicine, Kaohsiung Veterans \\ General Hospital, Kaohsiung 81362, Taiwan \\ 2 Research Center of Medical Informatics, Kaohsiung Veterans General Hospital, Kaohsiung 81362, Taiwan \\ 3 Department of Internal Medicine, Kaohsiung Municipal United Hospital, Kaohsiung 80457, Taiwan \\ 4 School of Medicine, National Yang-Ming University, Taipei 11221, Taiwan \\ 5 Yuh-Ing Junior College of Health Care and Management, Kaohsiung 80776, Taiwan \\ 6 Department of Pediatrics, Kaohsiung Veterans General Hospital, Kaohsiung 81362, Taiwan \\ 7 Department of Otolaryngology, Head and Neck Surgery, Kaohsiung Veterans General Hospital, \\ Kaohsiung 81362, Taiwan \\ 8 Institute of Hospital and Health Care Administration, National Yang-Ming University, Taipei 11221, Taiwan \\ 9 Department of Stomatology, Kaohsiung Veterans General Hospital, Kaohsiung 81362, Taiwan \\ 10 Department of Marine Biotechnology and Resources, National Sun Yat-sen University, \\ Kaohsiung 80424, Taiwan \\ 11 Department of Dental Technology, Shu-Zen Junior College of Medicine and Management, \\ Kaohsiung 82144, Taiwan \\ * Correspondence: tsujenkuo@gmail.com; Tel.: +886-7-346-8214; Fax: +886-7-346-8392
}

Received: 1 April 2020; Accepted: 21 April 2020; Published: 23 April 2020

\begin{abstract}
Background: Children with cerebral palsy (CP) place a considerable burden on medical costs and add to an increased number of inpatient days in Taiwan. Continuity of care (COC) has not been investigated in this population thus far. Materials and Methods: We designed a retrospective population-based cohort study using Taiwan's National Health Insurance Research Database. Patients aged 0 to 18 years with $\mathrm{CP}$ catastrophic illness certificates were enrolled. We investigated the association of COC index (COCI) with medical costs and inpatient days. We also investigated the possible clinical characteristics affecting the outcome. Results: Over five years, children with CP with low COCI levels had higher medical costs and more inpatient days than did those with high COCI levels. Younger age at $\mathrm{CP}$ diagnosis, more inpatient visits one year before obtaining a catastrophic illness certificate, pneumonia, and nasogastric tube use increased medical expenses and length of hospital stay. Conclusions: Improving COC reduces medical costs and the number of inpatient days in children with CP. Certain characteristics also influence these outcomes.
\end{abstract}

Keywords: continuity of care; children with cerebral palsy; medical costs; inpatient days

\section{Introduction}

Cerebral palsy (CP), the most common childhood-onset and permanent physical disability in numerous countries, is characterized by a neurodevelopmental disorder that affects muscle tone, movement, and motor skills. By international consensus in 2005, CP describes a group of permanent disorders of the development of movement and posture causing activity limitation, that are attributed to non-progressive disturbances that occurred in the developing fetal or infant brain. CP affects approximately 1 in 500 newborns with an approximate prevalence of 17 million people worldwide [1-3]. We applied the Gross Motor Function Classification System (GMFCS) as the gold standard to classify 
motor function in children with cerebral palsy. The levels of GMFCS have been described as follows: I: Children perform gross motor skills such as running and jumping, but speed, balance and coordination are limited; II: Children have only minimal ability to perform gross motor skills such as running and jumping; III: Children use wheeled mobility when travelling long distances and may self-propel for shorter distances; IV: children are transported in a manual wheelchair or use powered mobility; V: Children are limited in their ability to maintain antigravity head and trunk postures and control leg and arm movements [2].

The average lifetime economic cost per patient with $\mathrm{CP}$ assessed by the US Centers for Disease Control (CDC) was US\$921,000 in 2003 [4]. A Dutch study reported that the annual cost of severe CP was $€ 40,265$ (US\$50,855) per child [5]. In South Korea, the total cost of rehabilitation treatment per patient with CP aged 0 to 6 and 7 to 18 years was $\$ 8,066,013$ (US\$7259) and $\$ 7,448,412$ (US\$6704), respectively [6]. A Danish report stated a lifetime CP cost of approximately $€ 860,000$ (US\$971,800) and $€ 800,000$ (US\$904,000) in men and women, respectively-which are higher than the US CDC estimate [7]. Moreover, these costs may be a minimum value because certain costs would not have been extracted from the database. Patients with $\mathrm{CP}$ are a large burden on hospitalization resources. Toyokawa et al. reported $20.6 \%$ of patients with CP aged $<20$ years had been admitted in 1 year (over 2012-2013) [8]. In Australia, Meehan, et al. reported that $53 \%$ of patients with CP had at least one same-day admission, and 46\% had one or more multiday admissions from 2008 to 2012 [9]. Furthermore, patients with concurrent epilepsy or moderate to severe motor impairments (GMFCS III-V) had even more admissions. Primary and secondary prevention measures may reduce unnecessary and resource-wasting hospital admissions.

"Doctor-shopping", implicating frequent attendances and switching of physicians, has become common because of easy accessibility to local clinics or hospitals in Taiwan [10]. Patients can select any physician freely. Taiwan does not possess a well-established referral system, leading to a large amount of waste with regard to medical expenditure and caregiving workforce. This phenomenon could lead to a discontinuity of care, which will reduce treatment quality and exacerbate disease status. Methods for alleviating the medical costs and resource consumption of $\mathrm{CP}$ need to be reappraised and re-evaluated.

Continuity of care (COC) comprises two core elements: care of an individual patient and care over time. It comprises three types of care: informational continuity, management continuity, and relational continuity. By applying COC, a trusting and responsible therapeutic association can be established between the patient and physician [11-13]. COC involves a continuous caring relationship between doctors and patients. COC has also been described as a "seamless service [14,15]". Moreover, higher $\mathrm{COC}$ is related to a stronger sense of satisfaction, higher quality of life, better mental health, and lower costs $[16,17]$.

The effect of COC on children with $\mathrm{CP}$ has not been investigated. Therefore, we designed a retrospective population-based cohort study to investigate the association of $\mathrm{COC}$, inpatient days, and medical costs among children with CP in Taiwan. Furthermore, we surveyed the possible characteristics relevant to these areas.

\section{Materials and Methods}

\subsection{Study Design and Data Extraction}

We retrospectively extracted clinical data from the Taiwan National Health Insurance (NHI) Research Database (NHIRD), a nationwide population-based administrative database of NHI, the beneficiaries of which include approximately $99.9 \%$ of the population (i.e., approximately 23 million residents) of Taiwan [18]. NHIRD includes registration files on demographic data, medical visits, physicians, inpatient days, medical costs, procedure codes, and diagnostic codes according to the International Classification of Diseases, Ninth Revision, Clinical Modification (ICD-9-CM). NHIRD 
validation studies have reported high accuracy of its data $[19,20]$. A study also reported real-world evidence from the Catastrophic Illness Patient Database using NHIRD data analysis [21,22].

The current study was approved by the Institutional Review Board of Kaohsiung Veterans General Hospital, Kaohsiung, Taiwan (VGHKS15-EM10-02). The board also waived the need for written informed consent because all personal identifying information used had been de-identified. Figure 1 shows the inclusion and exclusion criteria. In total, 4496 children with CP (age at diagnosis 0-18 years) were identified between 1 January 2000, and 31 December 2012, based on ICD-9-CM code 343.X and catastrophic illness certificates. Patients were followed until 31 December 2013. The index date was set as the day the patients obtained the catastrophic illness certificate, confirmed by specialists in relevant fields. Medical service expenditures exhibited minor changes over the years. An exchange rate of US $\$ 1$ $=\mathrm{NT} \$ 30$ was used in our study-close to the current exchange rate.

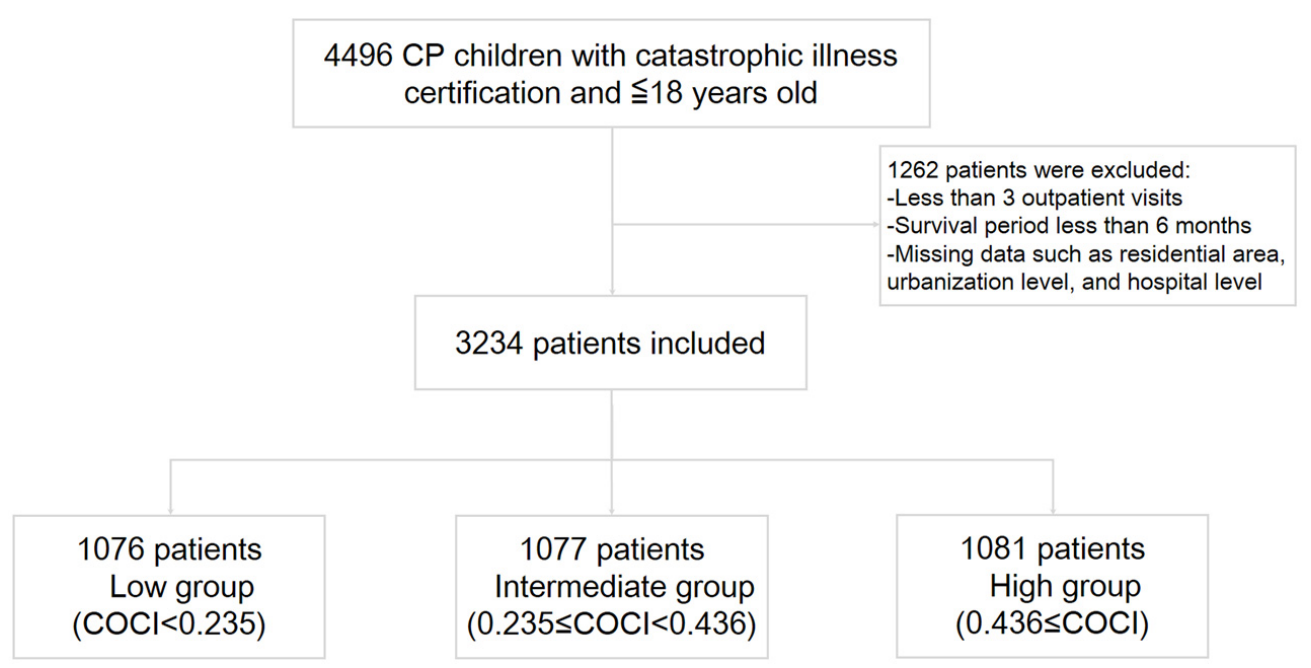

Figure 1. Recruitment of children diagnosed as having CP with catastrophic illness certification. Between 2000 to 2012, patients with low $(n=1076)$, intermediate COCI $(n=1077)$, and high $(n=1081)$ COCI. $\mathrm{CP}=$ cerebral palsy; $\mathrm{COCI}=$ continuity of care index.

\subsection{Patient Demographics and Characteristics}

Outpatient records were traced from the index date. Data were collected regarding outpatient visits one year after the index date, different physicians one year after the index date, age on the index date, gender, inpatient days over five years after the index date, medical costs over five years after the index date, inpatient visits one year before the index date, residential area, urbanization level, hospital level where children with CP obtained the catastrophic illness certificates, comorbidities, and mortality. The residential areas were classified as Northern, Central, and Southern-Eastern Taiwan. Urbanization level was dichotomized into urban and nonurban. Hospital levels, where children with $\mathrm{CP}$ obtained their catastrophic illness certificates, were categorized as medical centers and regional or district hospitals. The comorbidities considered in this research were asthma (ICD-9-CM 493), preterm labor (PTL) and small for gestational age (SGA; ICD-9-CM 765-765.19), perinatal complications (ICD-9-CM 760-764, 766-779, and V137), epilepsy (ICD-9-CM 345), pneumonia (ICD-9-CM 480-486, and 507.0-507.8), gastroesophageal reflux disease (GERD; ICD-9-CM 530.85, 530.11, and 530.81), and nasogastric tube use (ICD-9-CM 47017C and 47018C).

\subsection{Study Variables}

We compared total medical costs and inpatient days with a different $\mathrm{COC}$ index (COCI) and other characteristics. 


\subsection{COCI Application}

We applied the COCI developed by Bice and Boxerman to evaluate the COC status [23]. Several studies have used this method for COC investigation, extracting data from health care claim databases [24-28]. By using the COCI method, we could acquire the distribution of CP outpatient visits to different physicians, and visit times to each physician from the index date. According to Bice and Boxerman [23], the COCI measures a patient's COC based on the total number of visits and physicians as follows:

$$
\text { COCI }=\frac{\sum_{i=1}^{k} n_{i}^{2}-T}{T(T-1)},
$$

where $T$ is the total number of $\mathrm{CP}$ outpatient visits (CP being the principal diagnosis), $n_{i}$ is the number of times the patient visited a pediatric specialist, and $k$ is the total number of pediatric specialists visited. In our study, the one-year COCI was applied to compare the medical costs and the inpatient days of children with $\mathrm{CP}$ over five years.

\subsection{Statistical Analysis}

The outcome variables of interest were medical costs and inpatient days over five years from the index date. Medical costs involved fees paid by both caregivers of patients and the NHI Bureau in Taiwan. The distribution of variables was described by the chi-squared test. The continuous variables were compared using a one-way analysis of variance. The relationship between medical costs and inpatient days, as well as other variables, was analyzed using multiple linear regression and residual analysis. Multiple linear regression was not appropriate because of unequal variance assumptions (Figures 2 and 3). The effect of each variable or characteristic on medical costs and inpatient days was assessed by the hierarchical generalized linear model (HGLM) using a hospital-level random-intercept model [29-31]. The intraclass correlation (ICC) was also calculated based on estimations on the null model [32]. A 2-tailed $p$ value of $<0.05$ was considered significant. All statistical analyses were performed using SPSS (version 20; SPSS Inc., Chicago, IL, USA) and SAS (version 9.4; SAS Institute, Inc., Cary, NC, USA).

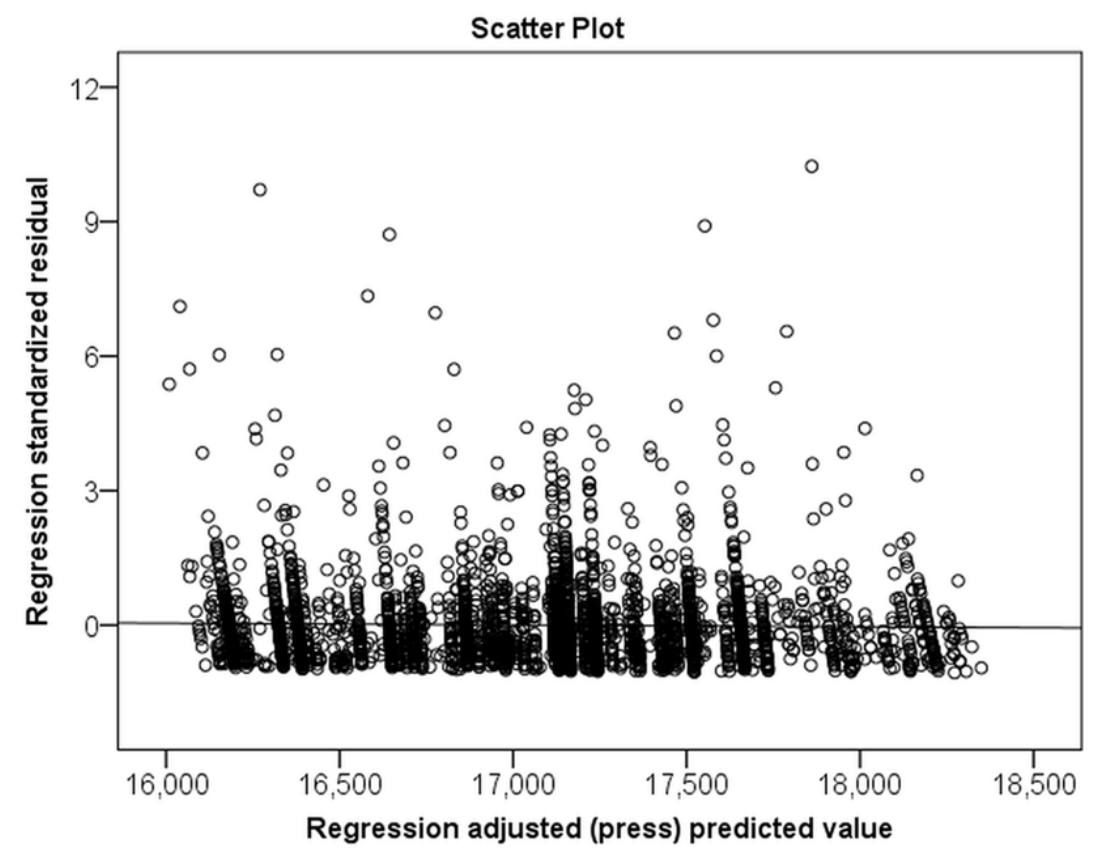

Figure 2. Residual plot illustrating that the standardized residual was not compatible with linear regression for medical costs over 5 years. 


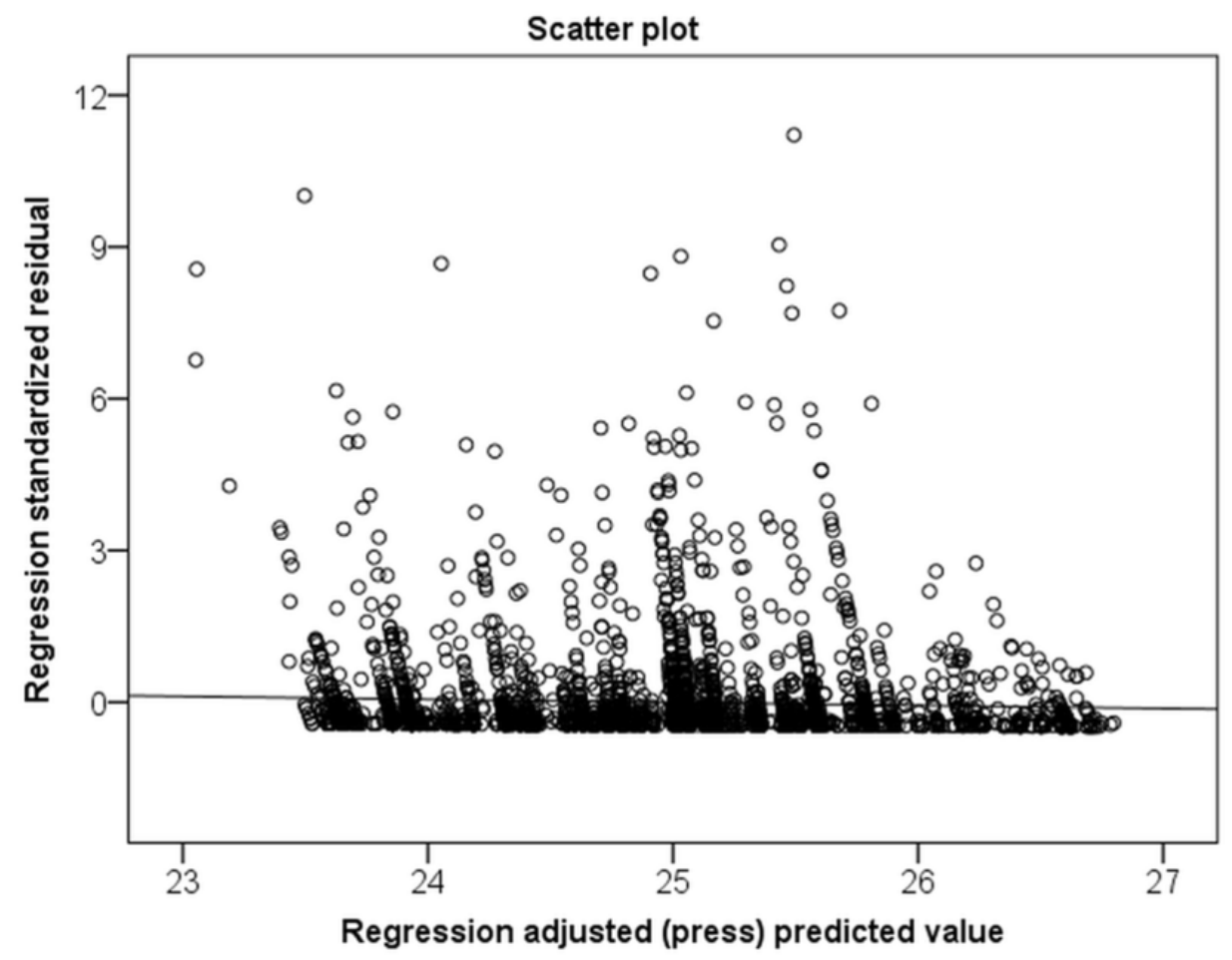

Figure 3. Residual plot illustrating that the standardized residual was not compatible with linear regression for numbers of inpatient days over 5 years.

\section{Results}

As illustrated in Figure 1, 3234 children with CP meeting our inclusion and exclusion criteria were identified from NHIRD. They were divided into three approximately equal groups according to patient numbers, the low (1076 patients, COCI < 0.235), intermediate (1077 patients, $0.235 \leq \mathrm{COCI}<0.436$ ), or high group (1081 patients, $0.436 \leq \mathrm{COCI})$. Table 1 summarizes basic demographic data, clinical characteristics, medical costs, inpatient days, comorbidities, and mortality. The top three reasons for admissions were pneumonia, other respiratory conditions except pneumonia and epilepsy (excluding ICD-9-CM codes of 343). Detailed ICD-9-CM codes were shown in File S1. The estimated ICC was 0.980 , indicating a significant clustering effect because the value was $>0.059$ [32].

\subsection{Univariate and Multivariate Analysis for Medical Costs}

The hospital-level random-intercept model HGLM, displayed in Table 2A,B, revealed that the average five-year medical costs for a child with $\mathrm{CP}$ in the low group were higher than for a child in the high COCI group in both univariate and multivariate analysis (US\$1656 more in multivariate analysis, $p=0.016$ ). The other characteristics associated with increased medical costs in both univariate and multivariate analysis were younger age, more inpatient visits, medical center, pneumonia, and nasogastric tube use. We performed a stratified analysis for medical costs after adjusting characteristics (Table 3A,B). The results indicated that the cost of five-year inpatient medical care was significantly higher in children with CP in the low group than in the high group (US\$1660 more in multivariate analysis, $p=0.002)$. 
Table 1. One-year continuity of care index categorized according to characteristics in children with cerebral palsy.

\begin{tabular}{|c|c|c|c|c|c|}
\hline & Total Patients & $\begin{array}{l}\text { Low Group } \\
(\mathrm{COCI}<0.235)\end{array}$ & $\begin{array}{l}\text { Intermediate Group } \\
(0.235 \leq \text { COCI }<0.436)\end{array}$ & $\begin{array}{l}\text { High Group } \\
(0.436 \leq \text { COCI })\end{array}$ & \\
\hline Characteristics & $\mathrm{n}=3234(\%)$ & $\mathrm{n}=1076(\%)$ & $\mathrm{n}=1077(\%)$ & $\mathrm{n}=1081(\%)$ & $p$-Value \\
\hline \multicolumn{6}{|l|}{ Outpatient visit } \\
\hline Mean (SD) & $20.1(12.2)$ & $19.6(12.5)$ & $21.8(13.2)$ & $18.7(13.6)$ & $<0.001$ \\
\hline Medium (Q1-Q3) & $17(10-27)$ & $17(10-27)$ & 19 (12-29) & $15(9-25)$ & $<0.001$ \\
\hline \multicolumn{6}{|l|}{ Physicians } \\
\hline Mean (SD) & $5.3(3.1)$ & $7.7(3.3)$ & $5.3(2.1)$ & $3.0(1.7)$ & $<0.001$ \\
\hline Medium (Q1-Q3) & $5(3-7)$ & $7.0(5-10)$ & $5.0(4-6)$ & $3.0(2-4)$ & $<0.001$ \\
\hline Age, mean (SD), years & $3.6(3.4)$ & $2.9(2.8)$ & $3.3(3.1)$ & $4.5(3.9)$ & $<0.001$ \\
\hline Gender (Male) & $1925(59.5)$ & $665(61.8)$ & $642(59.6)$ & $618(57.2)$ & 0.090 \\
\hline Inpatient days, mean (SD) & $24.9(55.6)$ & $31.6(64.5)$ & $24.2(52.7)$ & $19.0(47.4)$ & $<0.001$ \\
\hline \multicolumn{6}{|l|}{$\begin{array}{l}\text { Total medical costs } \\
\text { (U.S. dollars) }\end{array}$} \\
\hline Inpatient, mean (SD) & $\begin{array}{c}5366 \\
(14,062)\end{array}$ & $\begin{array}{c}6836 \\
(16,735)\end{array}$ & $\begin{array}{c}4977 \\
(12,210)\end{array}$ & $\begin{array}{c}4290 \\
(12,697)\end{array}$ & $<0.001$ \\
\hline Outpatient, mean (SD) & $\begin{array}{l}11,701 \\
(9309)\end{array}$ & $\begin{array}{l}12,353 \\
(9263)\end{array}$ & $\begin{array}{l}12,091 \\
(9266)\end{array}$ & $\begin{array}{l}10,663 \\
(9309)\end{array}$ & $<0.001$ \\
\hline Inpatient visits, mean (SD) & $1.2(1.7)$ & $1.3(1.8)$ & $1.3(1.8)$ & $1.0(1.6)$ & $<0.001$ \\
\hline Residential area & & & & & 0.028 \\
\hline Northern & 1679 (51.9) & $568(52.8)$ & $571(53.0)$ & $540(50.0)$ & \\
\hline Central & $720(22.3)$ & $223(20.7)$ & $261(24.2)$ & $236(21.8)$ & \\
\hline Southern/Eastern & $835(25.8)$ & $285(26.5)$ & $245(22.7)$ & $305(28.2)$ & \\
\hline Urbanization level & & & & & 0.539 \\
\hline Urban & $1912(59.1)$ & $637(59.2)$ & $649(60.3)$ & $626(57.9)$ & \\
\hline Non-urban & $1322(40.9)$ & $439(40.8)$ & $428(39.7)$ & $455(42.1)$ & \\
\hline Hospital level & & & & & 0.657 \\
\hline Medical center & $2055(63.5)$ & $673(62.5)$ & $694(64.4)$ & $688(63.6)$ & \\
\hline $\begin{array}{l}\text { Regional/District hospital } \\
\text { Comorbidity }\end{array}$ & 1179 (36.5) & $403(37.5)$ & $383(35.6)$ & $393(36.4)$ & \\
\hline Asthma & $450(13.9)$ & $136(12.6)$ & $162(15.0)$ & $152(14.1)$ & 0.269 \\
\hline PTL \& SGA & $1194(36.9)$ & 449 (41.7) & $393(36.5)$ & $352(32.6)$ & $<0.001$ \\
\hline Perinatal complication & $1873(57.9)$ & $676(58.4)$ & $629(58.4)$ & $568(52.5)$ & $<0.001$ \\
\hline Epilepsy & $1638(50.6)$ & $509(47.3)$ & $572(53.1)$ & $557(51.5)$ & 0.021 \\
\hline Pneumonia & 2051 (63.4) & $732(68.0)$ & $699(64.9)$ & $620(57.4)$ & $<0.001$ \\
\hline GERD & $201(6.2)$ & $88(8.2)$ & $66(6.1)$ & $47(4.3)$ & 0.001 \\
\hline Nasogastric tube use & 451 (13.9) & $169(15.7)$ & $142(13.2)$ & $140(13.0)$ & 0.123 \\
\hline Mortality & $568(17.6)$ & $203(18.9)$ & $192(17.8)$ & $173(16.0)$ & 0.209 \\
\hline
\end{tabular}

Abbreviations: COCI, Continuity of Care Index; SD, standard deviation; Q1-Q3, 1st-3rd quantile; PTL \& SGA, preterm labor and small for gestational age; GERD, gastroesophageal reflux disease.

Table 2. (A) Medical costs over 5 years in children with cerebral palsy categorized by the level of continuity of care index and analyzed by the univariate hierarchical generalized linear model using a hospital-level random-intercept model. (B) Medical costs over 5 years in children with cerebral palsy categorized by the level of continuity of care index and analyzed by the multivariate hierarchical generalized linear model using a hospital-level random-intercept model.

\begin{tabular}{|c|c|c|}
\hline \multicolumn{3}{|c|}{ (A) } \\
\hline Parameter & Estimated Costs (U.S. Dollars) & $p$-Value \\
\hline COCI group & & \\
\hline Low group $(\mathrm{COCI}<0.235)$ & 3571 & $<0.001$ \\
\hline $\begin{array}{l}\text { Intermediate group } \\
(0.235 \leq \mathrm{COCI}<0.436)\end{array}$ & 1952 & 0.006 \\
\hline High group $(0.436 \leq \mathrm{COCI})$ & Reference & \\
\hline \multicolumn{3}{|l|}{ Age, year } \\
\hline$\leq 2.5$ & 7876 & $<0.001$ \\
\hline$>2.5$ & Reference & \\
\hline
\end{tabular}


Table 2. Cont.

\begin{tabular}{|c|c|c|}
\hline \multicolumn{3}{|c|}{ (A) } \\
\hline Parameter & Estimated Costs (U.S. Dollars) & $p$-Value \\
\hline \multicolumn{3}{|l|}{ Gender } \\
\hline Male & 402 & 0.500 \\
\hline Female & Reference & \\
\hline \multicolumn{3}{|l|}{ Inpatient visits } \\
\hline $0-1$ & -7828 & $<0.001$ \\
\hline$>1$ & Reference & \\
\hline \multicolumn{3}{|l|}{ Residential area } \\
\hline Northern & -873 & 0.266 \\
\hline Central & -673 & 0.477 \\
\hline Southern/Eastern & Reference & \\
\hline \multicolumn{3}{|l|}{ Urbanization level } \\
\hline Urban & -168 & 0.782 \\
\hline Non-urban & Reference & \\
\hline \multicolumn{3}{|l|}{ Hospital level } \\
\hline Medical center & 2213 & 0.001 \\
\hline Regional/District & Reference & \\
\hline \multicolumn{3}{|l|}{ Comorbidity } \\
\hline \multicolumn{3}{|l|}{ Asthma } \\
\hline No & 129 & 0.879 \\
\hline Yes & Reference & \\
\hline \multicolumn{3}{|l|}{ PTL \& SGA } \\
\hline No & -410 & 0.501 \\
\hline Yes & Reference & \\
\hline \multicolumn{3}{|l|}{ Perinatal complication } \\
\hline No & -2839 & $<0.001$ \\
\hline Yes & Reference & \\
\hline \multicolumn{3}{|l|}{ Epilepsy } \\
\hline No & -1624 & 0.006 \\
\hline Yes & Reference & \\
\hline \multicolumn{3}{|l|}{ Pneumonia } \\
\hline No & -6049 & $<0.001$ \\
\hline Yes & Reference & \\
\hline \multicolumn{3}{|l|}{ GERD } \\
\hline No & -5216 & $<0.001$ \\
\hline Yes & Reference & \\
\hline \multicolumn{3}{|l|}{ Nasogastric tube use } \\
\hline No & $-10,069$ & $<0.001$ \\
\hline Yes & Reference & \\
\hline \multicolumn{3}{|c|}{ (B) } \\
\hline Parameter & Estimated Costs (U.S. Dollars) & $p$-Value \\
\hline \multicolumn{3}{|l|}{ COCI group } \\
\hline Low group $(\mathrm{COCI}<0.235)$ & 1656 & 0.016 \\
\hline $\begin{array}{l}\text { Intermediate group } \\
(0.235 \leq \mathrm{COCI}<0.436)\end{array}$ & -36 & 0.958 \\
\hline High group $(0.436 \leq \mathrm{COCI})$ & Reference & \\
\hline \multicolumn{3}{|l|}{ Age, year } \\
\hline$\leq 2.5$ & 5501 & $<0.001$ \\
\hline$>2.5$ & Reference & \\
\hline \multicolumn{3}{|l|}{ Gender } \\
\hline Male & -137 & 0.809 \\
\hline Female & Reference & \\
\hline
\end{tabular}


Table 2. Cont.

\begin{tabular}{|c|c|c|}
\hline \multicolumn{3}{|c|}{ (B) } \\
\hline Parameter & Estimated Costs (U.S. Dollars) & $p$-Value \\
\hline \multicolumn{3}{|l|}{ Inpatient visits } \\
\hline $0-1$ & -3784 & $<0.001$ \\
\hline$>1$ & Reference & \\
\hline \multicolumn{3}{|l|}{ Residential area } \\
\hline Northern & -731 & 0.285 \\
\hline Central & -178 & 0.824 \\
\hline Southern/Eastern & Reference & \\
\hline \multicolumn{3}{|l|}{ Urbanization level } \\
\hline Urban & -136 & 0.820 \\
\hline Non-urban & Reference & \\
\hline \multicolumn{3}{|l|}{ Hospital level } \\
\hline Medical center & 1173 & 0.044 \\
\hline Regional/District & Reference & \\
\hline \multicolumn{3}{|l|}{ Comorbidity } \\
\hline \multicolumn{3}{|l|}{ Asthma } \\
\hline No & -679 & 0.412 \\
\hline Yes & Reference & \\
\hline \multicolumn{3}{|l|}{ PTL \& SGA } \\
\hline No & 380 & 0.600 \\
\hline Yes & Reference & \\
\hline \multicolumn{3}{|l|}{ Perinatal complication } \\
\hline No & -1187 & 0.088 \\
\hline Yes & Reference & \\
\hline \multicolumn{3}{|l|}{ Epilepsy } \\
\hline No & 285 & 0.637 \\
\hline Yes & Reference & \\
\hline \multicolumn{3}{|l|}{ Pneumonia } \\
\hline No & -2513 & $<0.001$ \\
\hline Yes & Reference & \\
\hline \multicolumn{3}{|l|}{ GERD } \\
\hline No & -1674 & 0.151 \\
\hline Yes & Reference & \\
\hline \multicolumn{3}{|l|}{ Nasogastric tube use } \\
\hline No & -6677 & $<0.001$ \\
\hline Yes & Reference & \\
\hline
\end{tabular}

\subsection{Univariate and Multivariate Analysis for Inpatient Days}

The hospital-level random-intercept model HGLM, displayed in Table 4A,B, revealed that the average five-year inpatient days for a child with $\mathrm{CP}$ in the low group were longer than for a child in the high COCI group in both univariate and multivariate analysis ( 8 days more in multivariate analysis, $p<0.001)$. The other characteristics associated with increased inpatient days in both univariate and multivariate analysis were younger age, more inpatient visits, living in Southern and Eastern Taiwan, no PTL or SGA, pneumonia, and nasogastric tube use. 
Table 3. (A) Stratified analysis of inpatient medical costs for children with cerebral palsy according to the level of continuity of care index analyzed by the hierarchical generalized linear model using a hospital-level random-intercept model. (B) Stratified analysis of outpatient medical costs for children with cerebral palsy according to the level of continuity of care index analyzed by the hierarchical generalized linear model using a hospital-level random-intercept model.

\begin{tabular}{|c|c|c|}
\hline \multicolumn{3}{|c|}{ (A) } \\
\hline Parameter & Adjusted Estimated Cost (U.S. Dollars) & $p$-Value \\
\hline \multicolumn{3}{|c|}{ (Inpatient medical costs within 5 years) } \\
\hline \multicolumn{3}{|c|}{ COCI group } \\
\hline Low group $(\mathrm{COCI}<0.235)$ & 1660 & 0.002 \\
\hline $\begin{array}{l}\text { Intermediate group } \\
(0.235<\mathrm{COCI}<0.436)\end{array}$ & -458 & 0.397 \\
\hline High group $(0.436 \leq \mathrm{COCI})$ & Reference & \\
\hline \multicolumn{3}{|c|}{ (B) } \\
\hline Parameter & Adjusted Estimated Cost (U.S. Dollars) & $p$-Value \\
\hline \multicolumn{3}{|c|}{ (Outpatient medical costs within 5 years) } \\
\hline \multicolumn{3}{|c|}{ COCI group } \\
\hline Low group $(\mathrm{COCI}<0.235)$ & 614 & 0.121 \\
\hline $\begin{array}{l}\text { Intermediate group } \\
(0.235 \leq \mathrm{COCI}<0.436)\end{array}$ & 583 & 0.138 \\
\hline High group $(0.436 \leq \mathrm{COCI})$ & Reference & \\
\hline
\end{tabular}

Adjusted characteristics: age, gender, inpatient visits, residential area, urbanization level, hospital level, and comorbidities.

Table 4. (A) Inpatient days over 5 years in children with cerebral palsy categorized by the level of continuity of care index and analyzed by the univariate hierarchical generalized linear model using a hospital-level random-intercept model. (B) Inpatient days over 5 years in children with cerebral palsy categorized by the level of continuity of care index and analyzed by the multivariate hierarchical generalized linear model using a hospital-level random-intercept model.

\begin{tabular}{ccc}
\hline & $(\mathbf{A})$ & \\
\hline Parameter & Estimated Days & $p$-Value \\
\hline COCI group & & $<0.001$ \\
Low group (COCI $<0.235)$ & 13 & 0.037 \\
Intermediate group & 5 & \\
$(0.235 \leq$ COCI $<0.436)$ & Reference & \\
High group $(0.436 \leq$ COCI $)$ & & $<0.001$ \\
Age, year & 19 & \\
$\leq 2.5$ & Reference & 0.974 \\
$>2.5$ & -1 & \\
Gender & & $<0.001$ \\
Male & Reference & \\
Female & -39 & 0.013 \\
Inpatient visits & & 0.016 \\
$0-1$ & Reference & \\
$>1$ & -7 &
\end{tabular}


Table 4. Cont.

\begin{tabular}{|c|c|c|}
\hline \multicolumn{3}{|c|}{ (A) } \\
\hline Parameter & Estimated Days & $p$-Value \\
\hline \multicolumn{3}{|l|}{ Urbanization level } \\
\hline Urban & -2 & 0.295 \\
\hline Non-urban & Reference & \\
\hline \multicolumn{3}{|l|}{ Hospital level } \\
\hline Medical center & 9 & 0.006 \\
\hline Regional/District hospital & Reference & \\
\hline \multicolumn{3}{|l|}{ Comorbidity } \\
\hline \multicolumn{3}{|l|}{ Asthma } \\
\hline No & -1 & 0.743 \\
\hline Yes & Reference & \\
\hline \multicolumn{3}{|l|}{ PTL \& SGA } \\
\hline No & 12 & $<0.001$ \\
\hline Yes & Reference & \\
\hline \multicolumn{3}{|l|}{ Perinatal complication } \\
\hline No & 1 & 0.913 \\
\hline Yes & Reference & \\
\hline \multicolumn{3}{|l|}{ Epilepsy } \\
\hline No & -17 & $<0.001$ \\
\hline Yes & Reference & \\
\hline \multicolumn{3}{|l|}{ Pneumonia } \\
\hline No & -32 & $<0.001$ \\
\hline Yes & Reference & \\
\hline \multicolumn{3}{|l|}{ GERD } \\
\hline No & -10 & $<0.001$ \\
\hline Yes & Reference & \\
\hline \multicolumn{3}{|l|}{ Nasogastric tube use } \\
\hline No & -69 & $<0.001$ \\
\hline Yes & Reference & \\
\hline \multicolumn{3}{|c|}{ (B) } \\
\hline Parameter & Estimated Days & $p$-Value \\
\hline \multicolumn{3}{|l|}{ COCI group } \\
\hline Low group $(\mathrm{COCI}<0.235)$ & 8 & $<0.001$ \\
\hline Intermediate group & -1 & 0.991 \\
\hline High group $(0.436 \leq \mathrm{COCI})$ & Reference & \\
\hline \multicolumn{3}{|l|}{ Age, year } \\
\hline$\leq 2.5$ & 7 & $<0.001$ \\
\hline$>2.5$ & Reference & \\
\hline \multicolumn{3}{|l|}{ Gender } \\
\hline Male & -1 & 0.505 \\
\hline Female & Reference & \\
\hline \multicolumn{3}{|l|}{ Inpatient visits } \\
\hline $0-1$ & -20 & $<0.001$ \\
\hline$>1$ & Reference & \\
\hline \multicolumn{3}{|l|}{ Residential area } \\
\hline Northern & -6 & 0.018 \\
\hline Central & -7 & 0.014 \\
\hline Southern/Eastern & Reference & \\
\hline \multicolumn{3}{|l|}{ Urbanization level } \\
\hline Urban & -1 & 0.528 \\
\hline Non-urban & Reference & \\
\hline \multicolumn{3}{|l|}{ Hospital level } \\
\hline Medical center & 4 & 0.070 \\
\hline Regional/District hospital & Reference & \\
\hline
\end{tabular}


Table 4. Cont.

\begin{tabular}{ccc}
\hline & (B) & \\
\hline Parameter & Estimated Days & $p$-Value \\
\hline Comorbidity & & \\
Asthma & -2 & 0.933 \\
No & Reference & \\
Yes & & \\
PTL \& SGA & 6 & 0.004 \\
No & Reference & \\
Yes & -1 & 0.865 \\
Perinatal complication & Reference & \\
No & -2 & 0.381 \\
Yes & Reference & \\
Epilepsy & -14 & \\
No & $<0.001$ \\
Yes & Reference & \\
Pneumonia & -3 & 0.390 \\
No & Reference & \\
Yes & -54 & $<0.001$ \\
No & Reference & \\
Yes & &
\end{tabular}

\section{Discussion}

This study is the first large scale population-based cohort study on COC application among children with CP using NHIRD. We demonstrated that a low COC was associated with an increased number of inpatient days and more medical costs among children with CP. Furthermore, outpatient medical costs were higher than inpatient.

No studies had been conducted on COCI among children with CP regarding medical costs and inpatient days. Directly comparing the cost-effectiveness of different treatments and interventions is challenging. A systematic review conducted by Shin, et al. reported that the diversity of the decision contexts and health care systems complicated examining cost-effectiveness. Prevention is a critical strategy for cost-effectiveness [33]. A study by Ranken Jordan Pediatric Bridge Hospital demonstrated that intensive care coordination, psychosocial therapy, family and caregiver empowerment, and transitional care might alleviate costs and unnecessary hospital stays among children with CP [34].

Currently, COCI related research has demonstrated that high COCI could reduce costs and the number of inpatient days. In Korea, a nationwide cohort study was conducted on 47,433 patients with new diagnoses of hypertension, diabetes, hypercholesterolemia, and complications of these diseases in 2003 and 2004. The results revealed that high COCI was correlated with fewer inpatient days and reduced inpatient and outpatient health care costs [35]. A study conducted by Chen et al. reported that higher $\mathrm{COCI}$ was associated with fewer hospitalizations and emergency department visits for patients with diabetes-related diseases. Patients with high COCI scores saved more on health care expenses than those with low COCI scores [36].

In the current study, lower COCI was associated with higher medical costs, particularly in ambulatory patients with CP. These findings indicated that changing physicians was correlated with a large increase in medical costs associated with outpatient visits, and, to a lesser extent, increased medical costs resulting from inpatient visits. The five-year emergency department visits after the index date of children with CP are shown in Table S1. One-year COCI did not affect five-year emergency department visits significantly. In addition, firstly, our outcome of medical costs includes outpatient, 
inpatient, and emergency department visits totally. Second, in Taiwan, the medical costs of emergency visits are much less than in most other countries worldwide. Therefore, we did not take the emergency department visits into account in our results. In many diseases, such as acute myocardial infarction, gastrointestinal bleeding, acute kidney injury, and respiratory distress syndrome, outpatient medical costs are often lower than inpatient medical costs [37-40]. Our stratified analysis revealed that the low COCI group had more inpatient medical costs than the high COCI group. These findings indicated that children with $\mathrm{CP}$ and low COCI might have poorer care quality. Therefore, increased medical expenditure on treatments and recovery would be required once they are admitted. The low COCI group also had an increased number of inpatient days than the high COCI group. This result indicated that changing doctors might influence treatment quality, which would prolong the duration of treatment required during hospitalization. In our study, low COCI led to medical resource waste and an increased number of inpatient days. Higher COCI is necessary to improve care quality and economic efficiency of the health care system.

In our multivariate analysis of the characteristics of children with $\mathrm{CP}$, younger age was associated with increased medical expenditure, which is similar to a study performed by Park et al. They reported that annual health care cost per person with $\mathrm{CP}$ decreased gradually with growing age before the age of 18 years [41]. Our findings also indicated that younger age was related to an increased number of inpatient days. However, Chiang reported that the mean lengths of hospital stay increased with growing age $(9.8,10.4$, and 12.8 days in patients aged $4-12.9,13-17.9$, and 18-32.9 years, respectively) [42]. Increased inpatient visits and obtaining the catastrophic illness certificate at medical centers are objectively correlated with higher medical costs because children with CP would be brought to the same medical center several times for outpatient or inpatient visits. They could then obtain the catastrophic illness certificate, diagnosed by specialists. An increased number of inpatient visits also correlated with an increased number of inpatient days, because patients with $\mathrm{CP}$ who had more inpatient visits might have more serious diseases or complications. Our study revealed fewer inpatient days among children with CP living in Northern and Central Taiwan than among those living in Southern and Eastern Taiwan. According to the study conducted by Li et al., the highest resource supplementation was in Northern Taiwan, followed by Central, Southern, and Eastern Taiwan [43]. The uneven distribution of medical resources suggests that children with CP living in Southern or Eastern areas would have more inpatient days because of an inadequate workforce and medical support. In children with $\mathrm{CP}$, respiratory diseases, including pneumonia episodes, are a principal cause of premature death and hospitalization [44]. Procedures, medicines, and the care-providing source involved in inpatient hospital stays lead to large medical expenses. Children with $\mathrm{CP}$, as well as attacks of pneumonia, would also have an increased number of inpatient days. This phenomenon accords with research by Meehan, et al., which reported that respiratory diseases accounted for a larger proportion of children with a higher level of CP severity (GMFCS III-V) than those with a lower level of severity (GMFCS I-II) and were associated with the number of inpatient days [9]. Notably, patients with CP without PTL and SGA in our study had a higher number of inpatient days than those with PTL and SGA. These findings were unexpected because a study had reported that babies who were small for gestation age were more likely to have prolonged hospital stays [45]. We hypothesized that children with CP and PTL or SGA had a higher mortality rate in early life. Therefore, the number of inpatient days might be reduced in the subsequent follow-up. Nasogastric tube use increased expenses and inpatient days. A study on the costs of gastrostomy (G)/gastrojejunostomy (GJ)-tube feeding in North America reported that the cost of care for a patient with CP needing a G/GJ-tube was on average US $\$ 37,232$, nearly 2.5 -fold the cost of care without a G/GJ tube (US\$15,004) [46]. Several limitations of nasogastric tube feeding for long term use have been reported, including recurrent pulmonary aspiration [47]. Therefore, nasogastric tube use may be related to an increased number of inpatient days. The results from prior studies were similar to ours.

There are some limitations to our study. First, the study was retrospective, and some confounding factors could not be extracted from the NHIRD, including the unrecorded costs of medication and 
treatments at patients' own expense. Second, CP severity is often categorized using the Gross Motor Function Classification System. However, we could not extract the degree of CP severity from NHIRD. Tonmukayakul's systematic review reported a strong relationship between CP severity and expenditure [48]. This limitation might reduce the comprehensive evaluation of medical costs on patients with CP. Third, we could not identify disease severity every time patients with $\mathrm{CP}$ were brought to doctors' clinics. Visits to clinics could have also been for mild conditions, such as the common cold or mild diarrhea. Caregivers may not take the patients to see the same doctor under these circumstances. This may have led to bias in COCI calculation. Fourth, the diagnosis codes may not correspond to true situations. There is a possibility that difference between admission and discharge diagnoses existed. If a patient was discharged with only one diagnosis of $\mathrm{CP}$, we could not track the real admission etiology [42].

\section{Conclusions}

This population-based cohort study demonstrated that high $\mathrm{COCI}$ levels in children with $\mathrm{CP}$ decreased medical costs and inpatient days. We suggest that a child with $\mathrm{CP}$ should not change pediatricians frequently, in order to decrease medical costs and inpatient days. Moreover, younger age, more inpatient visits, residential area, hospital level, PTL and SGA, pneumonia, and nasogastric tube use exhibited an effect on medical costs and inpatient days. For policy-making and cost-effectiveness regarding children with $\mathrm{CP}$, further long-term and high-quality clinical studies should be conducted.

Supplementary Materials: The following are available online at http://www.mdpi.com/1660-4601/17/8/2913/s1, File S1: ICD-9-CM codes for inpatients, Table S1: Emergency department visits of children with CP.

Author Contributions: Conceptualization: K.-T.Y., T.-J.K., Y.-M.H., S.-J.H. Data curation: K.-T.Y., C.-H.Y., T.-J.K., S.-J.H. Formal analysis: K.-T.Y., C.-H.Y., T.-J.K. Investigation: K.-T.Y., T.-J.K. Methodology: K.-T.Y., C.-H.Y., T.-J.K., Y.-M.H., C.-C.L. Project administration: K.-T.Y., C.-H.Y., T.-J.K., C.-C.L. Visualization: K.-T.Y., C.-H.Y., T.-J.K., Y.-M.H. Writing-original draft: K.-T.Y. Writing-review \& editing: T.-J.K., Y.-M.H. All authors have read and agreed to the published version of the manuscript.

Funding: This research received no external funding.

Acknowledgments: The authors expressed their appreciation to the Department of Medical Education and Research and Research Center of Medical Informatics in Kaohsiung Veterans General Hospital for inquiries and assistance in data processing. This study was supported by grants from Kaohsiung Veterans General Hospital, Kaohsiung, Taiwan (VGHKS109-D04-1). This manuscript was edited by Wallace Academic Editing.

Conflicts of Interest: The authors declare no conflict of interest.

\section{References}

1. Gulati, S.; Sondhi, V. Cerebral palsy: An overview. Indian J. Pediatr. 2018, 85, 1006-1016. [CrossRef] [PubMed]

2. Graham, H.K.; Rosenbaum, P.; Paneth, N.; Dan, B.; Lin, J.-P.; Damiano, D.L.; Becher, J.G.; Gaebler-Spira, D.; Colver, A.; Reddihough, D.S.; et al. Cerebral palsy. Nat. Rev. Dis. Primers. 2016, 2, 15082. [CrossRef] [PubMed]

3. Güçhan, Z.; Mutlu, A. The effectiveness of taping on children with cerebral palsy: A systematic review. Dev. Med. Child Neurol. 2017, 59, 26-30. [CrossRef] [PubMed]

4. Centers for Disease Control Prevention. Economic costs associated with mental retardation, cerebral palsy, hearing loss, and vision impairment-United States, 2003. MMWR Morb. Mortal. Wkly. Rep. 2004, 53, 57-59.

5. Hoving, M.A.; Evers, S.M.A.A.; Ament, A.J.H.A.; van Raak, E.P.M.; Vles, J.S.H.; Dutch Group on Child Spasticity. Intractable spastic cerebral palsy in children: A Dutch cost of illness study. Dev. Med. Child Neurol. 2007, 49, 397-398. [CrossRef]

6. Kim, S.W.; Jeon, H.R.; Youk, T.; Kim, J. Cost of rehabilitation treatment of patients with cerebral palsy in Korea. Ann. Rehabil. Med. 2018, 42, 722-729. [CrossRef]

7. Kruse, M.; Michelsen, S.I.; Flachs, E.M.; Brønnum-Hansen, H.; Madsen, M.; Uldall, P. Lifetime costs of cerebral palsy. Dev. Med. Child Neurol. 2009, 51, 622-628. [CrossRef]

8. Toyokawa, S.; Maeda, E.; Kobayashi, Y. Estimation of the number of children with cerebral palsy using nationwide health insurance claims data in Japan. Dev. Med. Child Neurol. 2017, 59, 317-321. [CrossRef] 
9. Meehan, E.; Freed, G.L.; Reid, S.M.; Williams, K.; Sewell, J.R.; Rawicki, B.; Reddihough, D.S. Tertiary paediatric hospital admissions in children and young people with cerebral palsy. Child Care Health Dev. 2015, 41, 928-937. [CrossRef]

10. Chen, T.J.; Chou, L.F.; Hwang, S.J. Patterns of ambulatory care utilization in Taiwan. BMC Health Serv. Res. 2006, 6, 54. [CrossRef]

11. Haggerty, J.L.; Reid, R.J.; Freeman, G.K.; Starfield, B.H.; Adair, C.E.; McKendry, R. Continuity of care: A multidisciplinary review. BMJ 2003, 327, 1219-1221. [CrossRef] [PubMed]

12. Saultz, J.W. Defining and measuring interpersonal continuity of care. Ann. Fam. Med. 2003, 1, $134-143$. [CrossRef] [PubMed]

13. Jee, S.H.; Cabana, M.D. Indices for continuity of care: A systematic review of the literature. Med. Care Res. Rev. 2006, 63, 158-188. [CrossRef] [PubMed]

14. Gulliford, M.; Naithani, S.; Morgan, M. What is 'continuity of care'? J. Health Serv. Res. Policy 2006, 11, 248-250. [CrossRef]

15. Bachrach, L.L. Continuity of care for chronic mental patients: A conceptual analysis. Am. J. Psychiatry 1981, 138, 1449-1456. [CrossRef]

16. Chen, Y.Y.; Hsieh, C.I.; Chung, K.P. Continuity of care, follow-up care, and outcomes among breast cancer survivors. Int. J. Environ. Res. Public Health 2019, 16, 3050. [CrossRef]

17. Bazemore, A.; Petterson, S.; Peterson, L.E.; Bruno, R.; Chung, Y.; Phillips, R.L., Jr. Higher primary care physician continuity is associated with lower costs and hospitalizations. Ann. Fam. Med. 2018, 16, 492-497. [CrossRef]

18. Lin, L.Y.; Warren-Gash, C.; Smeeth, L.; Chen, P.C. Data resource profile: The National Health Insurance Research Database (NHIRD). Epidemiol. Health 2018, 40, e2018062. [CrossRef]

19. Cheng, C.L.; Chien, H.C.; Lee, C.H.; Lin, S.J.; Yang, Y.H.K. Validity of in-hospital mortality data among patients with acute myocardial infarction or stroke in National Health Insurance Research Database in Taiwan. Int. J. Cardiol. 2015, 201, 96-101. [CrossRef]

20. Hsieh, C.Y.; Chen, C.H.; Li, C.Y.; Lai, M.L. Validating the diagnosis of acute ischemic stroke in a National Health Insurance claims database. J. Formos. Med. Assoc. 2015, 114, 254-259. [CrossRef]

21. Chien, H.C.; Kao Yang, Y.H.; Bai, J.P.F. Trastuzumab-related cardiotoxic effects in Taiwanese women: A nationwide cohort study. JAMA Oncol. 2016, 2, 1317-1325. [CrossRef] [PubMed]

22. Hsieh, C.Y.; Su, C.C.; Shao, S.C.; Sung, S.F.; Lin, S.J.; Kao Yang, Y.H.; Lai, E.C.C. Taiwan's National Health Insurance Research Database: Past and future. Clin. Epidemiol. 2019, 11, 349-358. [CrossRef] [PubMed]

23. Bice, T.W.; Boxerman, S.B. A quantitative measure of continuity of care. Med. Care 1977, 15, 347-349. [CrossRef] [PubMed]

24. Chen, C.C.; Tseng, C.H.; Cheng, S.H. Continuity of care, medication adherence, and health care outcomes among patients with newly diagnosed type 2 diabetes: A longitudinal analysis. Med. Care 2013, 51, 231-237. [CrossRef] [PubMed]

25. $\mathrm{Pu}, \mathrm{C}$;; Chou, Y.J. The impact of continuity of care on emergency room use in a health care system without referral management: An instrumental variable approach. Ann. Epidemiol. 2016, 26, 183-188. [CrossRef]

26. Jang, Y.J.; Choy, Y.S.; Nam, C.M.; Moon, K.T.; Park, E.C. The effect of continuity of care on the incidence of end-stage renal disease in patients with newly detected type 2 diabetic nephropathy: A retrospective cohort study. BMC Nephrol. 2018, 19, 127. [CrossRef]

27. Lee, S.A.; Choi, D.W.; Kwon, J.; Lee, D.W.; Park, E.C. Association between continuity of care and type 2 diabetes development among patients with thyroid disorder. Medicine 2019, 98, e18537. [CrossRef]

28. Kuo, T.J.; Wu, P.C.; Tang, P.L.; Yin, C.H.; Chu, C.H.; Hung, Y.M. Effects of continuity of care on the postradiotherapy survival of working-age patients with oral cavity cancer: A nationwide population-based cohort study in Taiwan. PLoS ONE 2019, 14, e0225635. [CrossRef]

29. Lee, C.C.; Chang, T.S.; Wu, C.J.; Yang, C.C.; Chen, P.C. Determinants of end-of-life expenditures in patients with oral cancer in Taiwan: A population-based study. PLOS ONE 2015, 10, e0126482. [CrossRef]

30. Diez-Roux, A.V. Bringing context back into epidemiology: Variables and fallacies in multilevel analysis. Am. J. Public Health 1998, 88, 216-222. [CrossRef]

31. Diez-Roux, A.V. Multilevel analysis in public health research. Annu. Rev. Public Health 2000, 21, 171-192. [CrossRef] [PubMed]

32. Muller, K. Statistical power analysis for the behavioral sciences. Technometrics 1989, 31, 499-500. [CrossRef] 
33. Shih, S.T.F.; Tonmukayakul, U.; Imms, C.; Reddihough, D.; Graham, H.K.; Cox, L.; Carter, R. Economic evaluation and cost of interventions for cerebral palsy: A systematic review. Dev. Med. Child Neurol. 2018, 60, 543-558. [CrossRef] [PubMed]

34. Howard, S.W.; Zhang, Z.; Buchanan, P.; Armbrecht, E.; Williams, C.; Wilson, G.; Hutchinson, J.; Pearson, L.; Ellsworth, S.; Byler, C.M.; et al. The effect of a comprehensive care transition model on cost and utilization for medically complex children with cerebral palsy. J. Pediatr. Health Care 2017, 31, 634-647. [CrossRef]

35. Shin, D.W.; Cho, J.; Yang, H.K.; Park, J.H.; Lee, H.; Kim, H.; Oh, J.; Hwang, S.; Cho, B.; Guallar, E. Impact of continuity of care on mortality and health care costs: A nationwide cohort study in Korea. Ann. Fam. Med. 2014, 12, 534-541. [CrossRef]

36. Chen, C.C.; Chen, S.H. Better continuity of care reduces costs for diabetic patients. Am. J. Manag. Care 2011, $17,420-427$.

37. Cowper, P.A.; Knight, J.D.; Davidson-Ray, L.; Peterson, E.D.; Wang, T.Y.; Mark, D.B.; Translate-Acs Investigators. Acute and 1-year hospitalization costs for acute myocardial infarction treated with percutaneous coronary intervention: Results from the TRANSLATE-ACS Registry. J. Am. Heart Assoc. 2019, 8, e011322. [CrossRef]

38. Campbell, H.E.; Stokes, E.A.; Bargo, D.; Logan, R.F.; Mora, A.; Hodge, R.; Gray, A.; James, M.W.; Stanley, A.J.; Everett, S.M.; et al. Costs and quality of life associated with acute upper gastrointestinal bleeding in the UK: Cohort analysis of patients in a cluster randomised trial. BMJ Open 2015, 5, e007230. [CrossRef]

39. Collister, D.; Pannu, N.; Ye, F.; James, M.; Hemmelgarn, B.; Chui, B.; Manns, B.; Klarenbach, S.; Alberta Kidney Disease, N. Health care costs associated with AKI. Clin. J. Am. Soc. Nephrol. 2017, 12, 1733-1743. [CrossRef]

40. Cheung, A.M.; Tansey, C.M.; Tomlinson, G.; Diaz-Granados, N.; Matté, A.; Barr, A.; Mehta, S.; Mazer, C.D.; Guest, C.B.; Stewart, T.E.; et al. Two-year outcomes, health care use, and costs of survivors of acute respiratory distress syndrome. Am. J. Respir. Crit. Care Med. 2006, 174, 538-544. [CrossRef]

41. Park, M.S.; Kim, S.J.; Chung, C.Y.; Kwon, D.G.; Choi, I.H.; Lee, K.M. Prevalence and lifetime healthcare cost of cerebral palsy in South Korea. Health Policy 2011, 100, 234-238. [CrossRef] [PubMed]

42. Chiang, K.L.; Huang, C.Y.; Fan, H.C.; Kuo, F.C. Prolonged length of stay for acute hospital admissions as the increasing of age: A nationwide population study for Taiwan's patients with cerebral palsy. Pediatr. Neonatol. 2019, 60, 74-82. [CrossRef] [PubMed]

43. Li, H.J.; Chen, C.Y.; Tsai, C.H.; Kuo, C.C.; Chen, K.H.; Chen, K.H.; Li, Y.C. Utilization and medical costs of outpatient rehabilitation among children with autism spectrum conditions in Taiwan. BMC Health Serv. Res. 2019, 19, 354. [CrossRef] [PubMed]

44. Blackmore, A.M.; Bear, N.; Blair, E.; Langdon, K.; Moshovis, L.; Steer, K.; Wilson, A.C. Predicting respiratory hospital admissions in young people with cerebral palsy. Arch. Dis. Child. 2018, 103, 1119-1124. [CrossRef] [PubMed]

45. Jacobsson, B.; Ahlin, K.; Francis, A.; Hagberg, G.; Hagberg, H.; Gardosi, J. Cerebral palsy and restricted growth status at birth: Population-based case-control study. BJOG 2008, 115, 1250-1255. [CrossRef] [PubMed]

46. Heyman, M.B.; Harmatz, P.; Acree, M.; Wilson, L.; Moskowitz, J.T.; Ferrando, S.; Folkman, S. Economic and psychologic costs for maternal caregivers of gastrostomy-dependent children. J. Pediatr. 2004, 145, 511-516. [CrossRef]

47. Eltumi, M.; Sullivan, P.B. Nutritional management of the disabled child: The role of percutaneous endoscopic gastrostomy. Dev. Med. Child Neurol. 1997, 39, 66-68. [CrossRef]

48. Tonmukayakul, U.; Shih, S.T.F.; Bourke-Taylor, H.; Imms, C.; Reddihough, D.; Cox, L.; Carter, R. Systematic review of the economic impact of cerebral palsy. Res. Dev. Disabil. 2018, 80, 93-101. [CrossRef]

(C) 2020 by the authors. Licensee MDPI, Basel, Switzerland. This article is an open access article distributed under the terms and conditions of the Creative Commons Attribution (CC BY) license (http://creativecommons.org/licenses/by/4.0/). 